\title{
Uber den Nachweis von Störungen im unteren Karbachtal bei Wangen im Allgäu mit Hilfe von Bodengasmessungen
}

\author{
Von Horst WeInHold, Kiel
}

Mit 1 Abbildung

\begin{abstract}
$\mathrm{Zusammenfassung:} \mathrm{Im} \mathrm{Bereich} \mathrm{des} \mathrm{unteren} \mathrm{Karbachtales} \mathrm{zwischen} \mathrm{Karsee,} \mathrm{Leupolz}$ und der Unteren Argen wurde in acht Profilen die Verteilung von $\mathrm{CO}_{2}$ und $\mathrm{CH}_{4}$ im Boden gemessen. Auf Grund sprunghafter Veränderung des Gasgehalts an verschiedenen Stellen sowie der unterschiedlichen Gaskonzentration wurden mehrere Störungen mit Orientierung der Hoch- und Tiefschollen ermittelt. Ähnlich wie für andere Senken des nordöstlichen Bodenseeraumes kann daraus gefolgert werden, daß neben fluviatiler Erosion und Eisschurf auch tektonische Vorgänge an der Formung des Landschaftsreliefs beteiligt waren.
\end{abstract}

$\mathrm{Summary}$ : Eight cross sections were studied in the region of the lower Karbach valley, between Karsee, Leupolz and the river Untere Argen, and the $\mathrm{CO}_{2}$ and $\mathrm{CH}_{4}$ distribution in the soil was measured. On the basis of rapid changes in the soil gas content and the different gas concentration at various spots several faults together with upthrown and downthrown blocks were located. It can thus be concluded that similar to other depressions in the northeastern Bodensee region both fluviatile and glacial erosion and tectonic events have caused the topographic relief.

\section{Einführung}

Die Landschaft des nordöstlichen Bodenseeraumes ist in eine Reihe von Höhenrücken und Senken gegliedert. Zwischen Friedrichshafen und Wangen sind das die Täler der Schussen, des Bollenbachs und des Karbachs mit den sie trennenden Hochgebieten. Für die Entstehung des Reliefs kommen verschiedene Faktoren in Betracht:

1. Exaration

Die nordnordöstliche Streichrichtung der Senken entspricht etwa der Schubrichtung der Eismassen des Rheingletschers in diesem Bereich.

2. Fluviatile und glazifluviatile Erosion

Terrassen und Ablagerungen von Flußschottern sind in den Niederungen und Talrändern zu finden.

3. Tektonische Senkung des Untergrundes

In der weiteren Umgebung wurden Verwerfungen mit gleichsinnigem Streichen wie die oben genannten Täler nachgewiesen und beschrieben (EINSELE \& SchönEnberg 1964, Volz 1959, GwinNer 1955, u. a.).

Nach ERNST (1968) stellt jedes Störungssystem eine Art Drainage für alle Tiefengase dar, die während der Diagenese, Metamorphose oder Lagerstättenbildung anfallen. Die Ausbißzonen dieser Störungen sollten sich deshalb durch erhöhte Gasgehalte von der ungestörten Umgebung abheben. Ziel der vorliegenden Untersuchungen war es, unter Anwendung der von ERNST (1968) beschriebenen Methode der Bodengasmessungen Störungen im Bereich des unteren Karbachtales nachzuweisen.

An dieser Stelle sei Herrn Dipl.-Geol. Reinkensmeier für die technische Beratung und Hilfe gedankt. 


\section{Zur Arbeitsmethode}

Die Bodengasmessungen wurden nach ERNST (1968) vorgenommen. An jedem Meßpunkt wurden $\mathrm{CH}_{4}$ und $\mathrm{CO}_{2}$ im aus dem Bohrloch abgesaugten Gasgemisch bestimmt. Zur $\mathrm{CH}_{4}$-Messung wurde das nach dem Interferometerprinzip arbeitende Meßgerät, Typ 18 der Firma Riken-Keiki, benutzt. Die $\mathrm{CO}_{2}$-Messungen erfolgten mit Dräger-Prüfgasröhrchen, Typ $1 \% \mathrm{CO}_{2}$.

Die Bohrlöcher hatten eine Tiefe von $1 \mathrm{~m}$ und $2 \mathrm{~cm}$ Durchmesser. Die Abstände der Meßpunkte betrugen in der Regel $25 \mathrm{~m}$. An bestimmten Stellen der Profile wurde die Distanz auf 10, bzw. 5 m verkürzt, um ein genaueres Bild der Meßkurve zu erhalten. Die Messungen wurden im November und Dezember vorgenommen, wodurch sekundäre Veränderungen der Gaskonzentration im Boden durch unterschiedliche landwirtschaftliche Nutzung vermieden werden sollten. Verfälschungen der Meßkurven ließen sich an einigen Stellen trotz Versatz der Meßpunkte nicht vermeiden. In der Abbildung sind sie entsprechend verzeichnet.

\section{Die geologische Situation}

Das Karbachtal stellt eine langgestreckte Nord-Süd gerichtete tertiäre Hohlform dar, welche sich über eine Schwelle bei Alttann nach Nordosten in das Wurzacher Becken fortsetzt (German et al. 1967). Sein nördlicher Teil wird bis zum Schweinberg südlich Karsee und Leupolz von einer Serie aus Randmoränen und eisrandnahen Sedimenten ausgefüllt. Soweit für die Oberflächenkartierung zugänglich, gehören sie der letzten Vereisung an (Weinhold 1974). Der südliche Teil ist bis zur Unteren Argen deutlich als Niederung ausgebildet. Von dort hebt die Hohlform flach nach Süden aus.

In beiden Seiten der Niederung sind Mergel und Sandsteine der Oberen Süßwassermolasse in kleinen Tobeln aufgeschlossen. Die tertiären Schichten werden von Grundmoräne und eisrandnahen Schmelzwasserschottern überdeckt. Lokal sind Reste von Randmoränen und Eisrandschwemmfächern erhalten (WeINHOLd 1974). Die Talfüllung setzt sich an der Oberfläche aus Auelehm, umgelagertem Hangschutt und Torf zusammen. Über die tatsächliche Eintiefung der Senke liegen nur Daten nördlich von Karsee vor (GERMAN et al. 1967). Anzeichen von Tektonik wurden im Karbachtal an einer Stelle, in einer Kiesgrube bei dem Gehöft Edenhaus, festgestellt. Dort ist eine kleine Störung mit Sprunghöhe von ca. 0,5 m aufgeschlossen.

\section{Auswertung der Meßprofile}

Die Gasgehalte der Profile 1 bis 8 reichen für $\mathrm{CO}_{2}$ von $\mathrm{O}$ bis maximal $6 \%$, für $\mathrm{CH}_{4}$ von $\mathrm{O}$ bis 2,6\%. Durchschnittlich pendeln sie bei $\mathrm{CO}_{2}$ zwischen 1 und $2 \%$, bei $\mathrm{CH}_{4}$ zwischen 0,1 und 1\%. Diese Werte entsprechen denen, welche auch ERNST bei seinen Untersuchungen in der ungefalteten Molasse erhielt.

Das Profil 1, nördlich des Gehöfts King beginnend und nach gut $850 \mathrm{~m}$ östlich der Straße Karsee-Pfaffenweiler endend (vgl. Lageplan), zeichnet sich durch eine verhältnismäßig ruhig verlaufende $\mathrm{CH}_{4}$-Kurve aus. Die Gehalte liegen unter 1\%. Die bewegtere $\mathrm{CO}_{2}$-Kurve schwankt zwischen durchschnittlich 1 bis $2 \% .250 \mathrm{~m}$ vom westlichen Endpunkt entfernt sticht ein kleiner Peak in beiden Gaskurven hervor, der durch eine Depression abgesetzt ist (infolge Bodenverdichtung konnte kein Gas abgepumpt werden). Der Peak - 3\% $\mathrm{CO}_{2}, 0,6 \% \mathrm{CH}_{4}$ - könnte unter Umständen eine Störung anzeigen, jedoch ist nach beiden Seiten kein Unterschied in der Gaskonzentration gegenüber dem bisherigen Kurvenverlauf festzustellen. Nach ERNST wäre auf der Hochscholle eine höhere Gasanreicherung als auf der Tiefscholle zu erwarten. 
--- $\mathrm{CO}_{2}$ Meßkurve

$-\mathrm{CH}_{4}$ MeAkurve

d Depression

」 Jauchedüngung

w Messungen im Grundwasserniveau

** ${ }^{*} \mathrm{M}$ Torf, Mudde

1,1, A Auelehm

$\mathrm{K}-\mathrm{H}$ Hangschutt

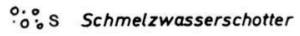

E Eisrandschwemmkegel

ง $\tilde{L} \mathrm{~L}_{\mathrm{R}}$ Randmoräne

:-.G Grundmoräne

ât: Tertiär (OSM)
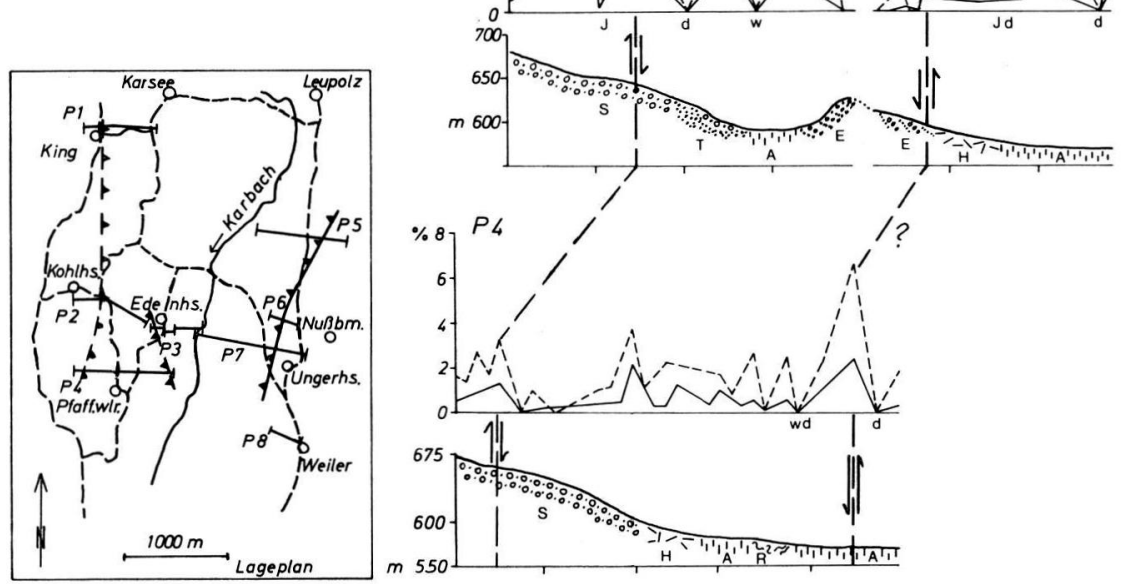

Abb. 1. Gasverteilungskurven von $\mathrm{CO}_{2}$ und $\mathrm{CH}_{4}$ an Meßprofilen im unteren Karbachtal bei Wangen/Allgäu. $\frac{\pi+1}{1200}$
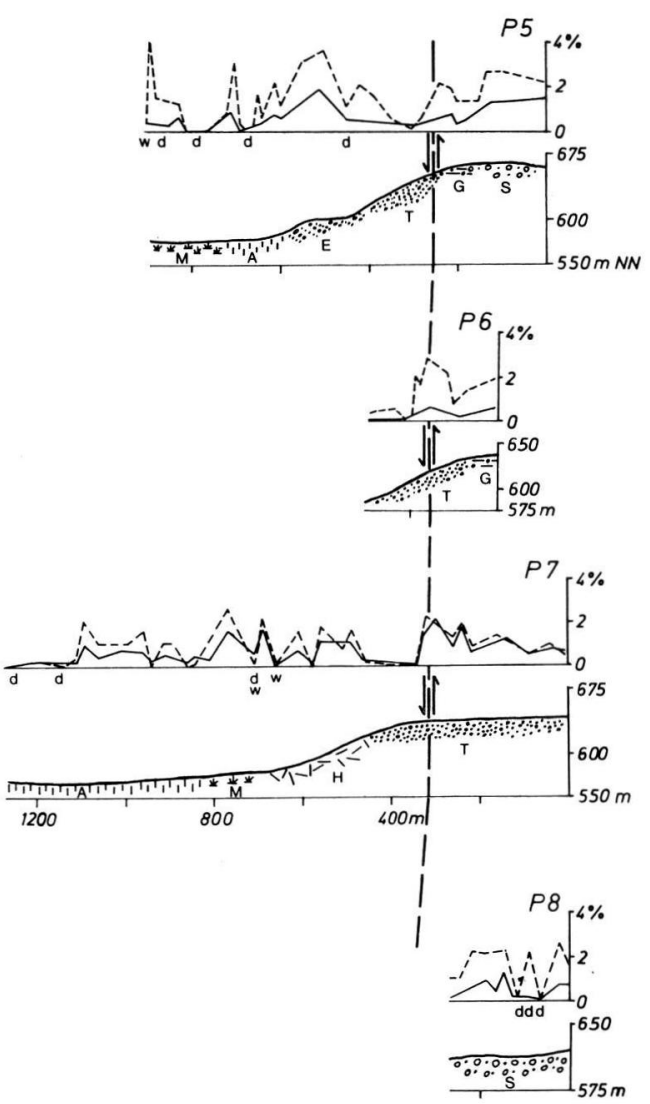
Das Profil 2 reicht von Kohlhaus bis westlich Edenhaus. Die Gasgehalte liegen allgemein über denen von Profil 1. Etwa $300 \mathrm{~m}$ vom westlichen Profilende entfernt, tritt wie bei Profil1 ein Gaspeak auf. Der $\mathrm{CO}_{2}$-Gehalt steigt auf $4 \%$, der $\mathrm{CH}_{4}$-Gehalt auf 2,6\% an. Der weitere Verlauf der Gaskurven wird durch eine Depression und hoch anstehendes Grundwasser unterbrochen. Mit Vorbehalt kann im Peak die Fortsetzung der vermuteten Störung von Profil 1 festgelegt werden. Das in kurzer Entfernung an Profil 2 anschließende Profil 3 besitzt mehr Aussagekraft. Deutlich ist der westliche Teil mit geringen Gasgehalten, $\mathrm{CO}_{2}$ bis $1 \%, \mathrm{CH}_{4}$ bis $0,2 \%$, von dem östlichen mit höheren, $\mathrm{CO}_{2}$ bis $4 \%$, $\mathrm{CH}_{4}$ bis $2 \%, \mathrm{zu}$ unterscheiden. Eine Störung ist auf gleicher Höhe des Profilpunktes, in dem die Gasgehalte ansteigen, nur $10 \mathrm{~m}$ weiter südlich in der oben erwähnten Kiesgrube bei Edenhaus aufgeschlossen. Die Hochscholle liegt auf der Ostseite, die Tiefscholle auf der Westseite, was sich mit den Gaskurven deckt.

Profil 4, nördlich Pfaffenweiler verlaufend, beginnt im Westen mit höheren Gasgehalten. Nach etwa $140 \mathrm{~m}$ sinkt die Gaskonzentration bis auf Spuren ab, um dann wieder anzusteigen. Die $\mathrm{CH}_{4}$-Kurve zeigt den Verlauf besonders gut. $600 \mathrm{~m}$ vor Ende des Profils ist die ursprüngliche Konzentration wieder erreicht $\left(\mathrm{CH}_{4}\right.$ zwischen 0,5 und $2 \%, \mathrm{CO}_{2}$ zwischen 1 und 3\%). Die in Profil 1 und 2 vermutete Störung tritt hier klar hervor. Am Ostende des Profils befindet sich ein markanter Gaspeak mit 6,6\% $\mathrm{CO}_{2}$ und 2,4 \% $\mathrm{CH}_{4}$. Zwar wird der Kurvenverlauf durch die benachbarten Depressionen abgewandelt, aber dennoch darf man in diesem Anstieg des Gasgehalts die Fortsetzung der Störung aus Profil 3 vermuten. Der abnehmende Gasgehalt westlich der Störung zeigt an, daß dort die Tiefscholle liegt. Profil 5 wird durch eine Reihe von Depressionen unterbrochen. Generell dürfte der Gasgehalt in der westlichen Hälfte des Profils aber höher als in der Osthälfte sein. Der plötzliche Anstieg der Meßkurven $250 \mathrm{~m}$ vor dem Ostende der Meßstrecke weist auf eine Störung hin, bei der sich die Hochscholle rechts, die Tiefscholle links befindet.

Das kurze Profil 6 nordwestlich Nußbaum erfaßt die Störung ebenfalls.

Im Westen überschneidet sich Profil 7 um $70 \mathrm{~m}$ mit Profil 3. Auf Grund der Depressionen ist der Verlauf der Gaskurven im Westen schwierig zu interpretieren. Der Gasgehalt scheint aber nach Osten geringer $\mathrm{zu}$ werden und bis gegen $0 \% \mathrm{zu}$ sinken. Im sprunghaften Anstieg der $\mathrm{CO}_{2}$ - und $\mathrm{CH}_{4}$-Werte auf $2 \%$ ist der Bereich der Störung zu erkennen. Gegen das Profilende nimmt der Gasgehalt langsam ab. Hoch- und Tiefscholle der Verwerfung sind wie in den Profilen 5 und 6 orientiert.

Profil 8 liegt außerhalb der Störungszone. Die Gasgehalte bleiben, abgesehen von den Drepressionen, ziemlich konstant.

\section{Ergebnisse der Messungen}

Mit Hilfe der Bodengasmessungen konnten auf beiden Seiten der Karbachsenke Störungen festgestellt werden. Im Westen verläuft eine vermutete Störung vom Gehöft King östlich an Kohlhaus vorbei in Nord-Süd-Richtung. Südlich Kohlhaus schwenkt sie nach Südwesten ab. Eine weitere Störung mit gegensinnigem Versatz führt von Edenhaus in südöstliche Richtung auf den Karbach zu. Auf dem Ostabhang der Niederung wurde eine dritte Störung von Ungerhaus in nordöstliche Richtung an Nußbaum vorbeiziehend ermittelt.

Die unterschiedliche Zusammensetzung und Konsistenz der Sedimente des Untergrundes hat scheinbar keinen meßbaren Einfluß auf die Verteilung der Bodengasmenge. In Bereichen von Stauchmoränen, Schmelzwasserschottern und Eisrandschwemmfächern, die eine bessere Durchlässigkeit haben als Grundmoränenmaterial und Tertiärsedimente, konnte 
kein Unterschied den Gasgehalt betreffend festgestellt werden. Ebensowenig treten Schwankungen an den Grenzen der einzelnen Sedimente auf.

In Profil 2 und 3 wurden Messungen auf Feldern vorgenommen, auf die zwei Tage zuvor Mist und Jauche gestreut wurde. Veränderungen in der Gaskonzentration des Bodens waren deswegen nicht zu beobachten.

\section{Lit e r a t u r}

Einsele, G. \& Schönenberg, R.: Epirogen-tektonische Grundzüge des süddeutschen Beckens und seiner südöstlichen Randgebiete im Mesozoikum. - Publ. Serv. geol. Luxemb. 14, 139-164, Luxembourg 1964.

ERnst, W.: Verteilung und Herkunft von Bodengasen in einigen süddeutschen Störungszonen. Erdöl u. Kohle 21, Teil 1, 605-610, Teil 2, 692-697, Hamburg 1968.

German, R., Lohr, J., Wittmann, D. \& Brosse, P.: Die Höhenlage der Schichtengrenze TertiärQuartär im mittleren Oberschwaben. - Eiszeitalter u. Gegenwart 18, 104-109, O'hringen/ Württ. 1967.

Gwinner, M. P.: Erdbebenherde und Tektonik in Oberschwaben. - N. Jb. Geol. Paläont. Mh., 475-478, Stuttgart 1955.

Volz, E.: Geologische Ergebnisse einiger Erdölbohrungen im westlichen Molassebecken. - Erdöl u. Kohle 12, 209-216, Hamburg 1959.

WeInHold, H.: Beiträge zur Kenntnis des Quartärs im württembergischen Allgäu zwischen östlichem Bodensee und Altdorfer Wald. - Diss. Tübingen 1974.

Manuskript eingeg. 7. 10. 1974.

Anschrift des Verf.: Dr. Horst Weinhold, Geologisches Landesamt von Schleswig-Holstein, 23 Kiel, Mercatorstraße 7. 